\title{
The Role of Microstructure and Processing on Magnetic Properties of Materials
}

\author{
M.A. WILLARD ${ }^{1,3}$ and V. FRANCO ${ }^{2,4}$ \\ 1.-Department of Materials Science and Engineering, Case Western Reserve University, \\ Cleveland, $\mathrm{OH}$ 44106, USA. 2.-Departamento de Física de la Materia Condensada, ICMSE-CSIC, \\ Universidad de Sevilla, 41080 Sevilla, Spain. 3.-e-mail: maw169@case.edu. 4.-e-mail: \\ vfranco@us.es
}

Magnetic materials can be viewed in light of their utility for the betterment of humanity, for underlying physics as a basis for new discovery, or simply for the wonderment of the phenomena that is magnetism. The practical application of our knowledge of magnetism and magnetic materials relies heavily on materials scientists and engineers. Principally, this is due to our understanding of the processingstructure-property relationships that are essential to making progress in development of improved performance. Specifically, the role of processing and its effect on microstructure evolution have significant impact on extrinsic magnetic properties, including coercivity, remanent magnetization, and permeability.

This optimization of (magnetic) properties by processing and control of the microstructure is deeply linked to the general interests of TMS, and there have been some specific activities addressing this topic; for instance, the symposium on Processing to Control Morphology and Texture in Magnetic Materials at the 2012 TMS annual meeting was one of the most recent. The following articles aim to provide a broad overview of some current trends in this field of research.

The materials science behind advances in heatassisted magnetic recording media is highlighted in the contribution from Varaprasad et al. This article describes continuing design and development of FePt alloys with refined, textured, and atomically ordered grains for the next generation of magnetic storage media. The need of each of the foregoing microstructural features is described with discussion of the important alloy design and processing challenges that allow their preparation.

The recent efforts to reduce reliance on critical materials have resulted in some interesting research in the field of magnetic materials. One of these topics is devoted to understanding and improving the rareearth free Alnico alloys. The contribution from
Palasyuk et al. describes work on the microstructure and magnetic domain structure of Alnico grades 5-7 and 9. The interplay of microstructure and domain structure is described through the analysis of domain images, revealing pinning mechanisms related to microstructural features.

Although soft magnetic nanocrystalline alloys are usually obtained by melt spinning and subsequent annealing, mechanical alloying has been shown as a very versatile "one-step" technique to produce nanocrystalline and amorphous metastable systems, including those compositions with interesting soft magnetic properties. The article by Blázquez et al. gives an overview of how studies of the dynamics of milling can be used to optimize the microstructure and magnetic properties of the obtained materials, stressing how the materials' properties depend on multiscale parameters (grain size versus crystal size).

Magnetic oxide materials are used in a wide range of high switching frequency (over $500 \mathrm{MHz}$ ) applications due in part to their large resistivities. Such applications include military and commercial radar, communication electronics, and passive electronics. The article by Harris et al. focuses on the role of magnetic, microstructure, and structural anisotropies in determining the radio-frequency $(\mathrm{RF})$ properties of the magnetic oxides with spinel, magnetoplumbite, and garnet structures. The important role of crystallographic texture used to modify the magnetic anisotropy enabling a tuning of the operating frequency, bandwidth, and loss, among other properties, is discussed.

Although these four previous articles rely on frequently used processing and microstructure evolution techniques, in this series of articles we also highlight some other possibilities of magnetic materials fabrication, which are not so familiar.

The first of these articles, by Torrejon et al., is devoted to the fabrication of multilayer magnetic microwires, in which a core of magnetic material 
(soft or hard) covered by an insulating Pyrex layer is fabricated by quenching and drawing, and it is subsequently electroplated with another layer of magnetic material (soft or hard). The control of the different parameters of this particular microstructure, which in turn modifies the coupling between magnetic phases, enables a rich phenomenology, which can be exploited in technological applications.

A completely different approach is presented by Rivoirard, who gives an overview on the processing of magnetic materials in static, intense magnetic fields. The energy terms appearing as the interaction of the material with these intense fields can give rise to a modification of the magnetic anisotropy of the processed material, alteration of the microstructure, and shifts of the transition temperatures of the different phases present in the sample.

We are convinced that this compilation of articles can contribute to bridging the gap between the larger metallurgy and materials community inside TMS and the still smaller subset of magneticians in this our society. Experts in materials processing can certainly take part in developing microstructures that will eventually enhance the properties of magnetic materials for current or future applications. At the same time, magnetic materials can provide a technologically important experimentation ground for testing processes for microstructure control and optimization. 